\title{
Stent-assisted coiling of wide-necked intracranial aneurysms using the Solitaire AB stent
}

\author{
Vikram Huded $^{1}$, Rithesh R. Nair ${ }^{1}$, Devashish D. Vyas ${ }^{2}$, Bhumir N. Chauhan ${ }^{2}$ \\ ${ }^{1}$ Division of Interventional Neurology and Stroke and ${ }^{2}$ Department of Neurology, Narayana Health Institute of Neurosciences, Bangalore, \\ Karnataka, India
}

\begin{abstract}
Context: Stent-assisted coiling of wide-necked and complex intracranial aneurysms is an effective and feasible treatment option. The self-expanding, fully retrievable Solitaire $A B\left(\mathrm{eV}_{3}\right.$, Irvine, $\left.\mathrm{CA}, \mathrm{USA}\right)$ stent is the latest neurovascular remodeling device available. To the best of our knowledge, there are no studies of Solitaire AB-assisted coiling of wide-necked intracranial aneurysms from India. Aim: Solitaire AB-assisted coiling of wide-necked intracranial aneurysms. Materials and Methods: The study was conducted in a tertiary care center with a dedicated Interventional Neurology division from 2009 to 2013. Consecutive patients with wide-necked aneurysms who underwent coiling assisted by the Solitaire $\mathrm{AB}$ stent were enrolled in the study. Axium $3 \mathrm{D}$ and Helix (eV 3 , Irvine, CA, USA) platinum coils were used to densely pack the aneurysm sac after deploying the stent across the neck. All patients were pretreated with antiplatelets according to protocol. Subsequently, dual antiplatelets were given for 6 months followed by continued aspirin. Outcome was assessed at 3 months using the modified Rankin Scale. Statistical Analysis Used: Statistical analysis was done using the SPSS 17.0 software. Results: A total of 16 patients underwent stent-assisted coiling. The most common site was the internal carotid artery (nine patients), median aneurysm size was $7 \mathrm{~mm}$ and median neck diameter was $5 \mathrm{~mm}$. Thirteen patients presented with ruptured aneurysms. We achieved complete occlusion in all patients with no major complications. Thirteen patients were followed up, all have an mRS score of zero or one. Conclusion: We conclude that for wide-necked aneurysms, stent-assisted coiling using the Solitaire AB is a safe and effective treatment option.
\end{abstract}

Key words: Aneurysm, coiling, intracranial, solitaire, stent-assisted

\section{Introduction}

Intracranial aneurysms can be treated either surgically by clipping or endovascularly by coiling. Endovascular coiling was validated as a treatment option in the International Subarachnoid Aneurysm Trial (ISAT). ${ }^{[1]}$ Since then, endovascular coiling has become a standard of care. However, aneurysms with wide necks or complex morphology pose a challenge to this method of treatment. Development of endovascular remodeling devices or stents has revolutionized management of such aneurysms. Of the various remodeling devices available, the Solitaire

\begin{tabular}{|l|l|}
\hline \multicolumn{2}{|c|}{ Access this article online } \\
\hline Quick Response Code: & Website: \\
\hline & www.ruralneuropractice.com \\
\cline { 2 - 2 } & \\
\hline & \\
\hline
\end{tabular}

$\mathrm{AB}$ self-expandable stent (eV3, Irvine, $\mathrm{CA}$, USA) is the latest one. ${ }^{[2]}$ We present our experience of stent-assisted coiling of wide-necked aneurysms in a tertiary care center from India. To the best of our knowledge, this is the first study from India using the Solitaire AB stent.

\section{Materials and Methods}

A database is maintained at our center for all patients undergoing endovascular procedures. The data were reviewed retrospectively from 2009 till date. Outpatient and inpatient records, procedural reports, and images were analyzed to determine anatomical and procedural details as well as adverse outcomes. All patients who were diagnosed with wide-necked aneurysms (neck diameter of $4 \mathrm{~mm}$ or more ${ }^{[2]}$ or dome: Neck ratio less than two) and underwent stent-assisted coiling were included.

All patients were premedicated with antiplatelets before the procedure. Patients who presented with unruptured

Address for correspondence:

Dr. Vikram Huded, Narayana Health Institute of Neurosciences, 258/A, Bommasandra Industrial Area, Hosur Main Road, Bangalore - 560 099, Karnataka, India. E-mail: drvikramhuded@gmail.com 
aneurysms were treated with aspirin $150 \mathrm{mg}$ and clopidogrel $75 \mathrm{mg}$ for 3 days before the procedure. Those patients who presented with subarachnoid hemorrhage were given $300 \mathrm{mg}$ of clopidogrel and $325 \mathrm{mg}$ of aspirin $2 \mathrm{~h}$ before the procedure. All procedures were done under general anesthesia and systemic heparinization. The right femoral percutaneous approach was used in all patients. A 3D angiogram was done to precisely define the configuration of the aneurysm. The Solitaire $\mathrm{AB}$ stent (eV3) was negotiated across the aneurysm neck using Rebar microcatheter (eV3). Another microcatheter was advanced into the aneurysm sac and jailed by deploying the stent. ${ }^{[3]}$ Intravenous abxicimab $5 \mathrm{mg}$ was given bolus after deployment of first two coils followed by intravenous infusion for $12 \mathrm{~h}$ in patients with recently ruptured aneurysm who developed filling defect in the stent during coiling. The aneurysm sac was then densely packed using platinum coils (Axium 3D and Helix; eV3). Postpacking angiogram was done to document exclusion of the aneurysm from the circulation. All patients were treated with aspirin $150 \mathrm{mg}$ and clopidogrel $75 \mathrm{mg}$ daily for 6 months and then continued on aspirin $150 \mathrm{mg}$ for life. Outcome was assessed at discharge and then at 3 months using the modified Rankin Scale, ${ }^{[4]}$ either by personal or telephonic interview. ${ }^{[5]}$ Control angiograms were done where feasible 6 months after the coiling procedure. SPSS version 17.0 software was used to analyze the data.

\section{Results}

\section{Patient characteristics}

A total of 16 patients underwent stent-assisted coiling of wide-necked aneurysms at our center between 2009 and 2013, of whom four were males and 12 females. The mean age at presentation was 51 years. Thirteen patients presented with ruptured aneurysms and subarachnoid hemorrhage, two presented with unruptured aneurysms and headache, and one presented with an unruptured aneurysm and cranial nerve palsy.

\section{Aneurysm size and locations}

The characteristics of the aneurysms are outlined in table 1.

Table 1: Characteristics of the aneurysms

\begin{tabular}{lc}
\hline Parameter & Details \\
\hline Morphology $(\mathrm{mm})$ & \\
$\quad$ Median size & 7 \\
Median neck diameter & 5 \\
Location & \\
Internal carotid artery & 9 \\
Middle cerebral artery & 2 \\
Posterior circulation & 5 \\
\hline
\end{tabular}

\section{Immediate results}

Stent deployment was successful in all the patients. Angiographic results included complete occlusion in all 16 patients $(100 \%)$. There were no neck remnants and no incomplete occlusions.

\section{Complications}

Four patients had minimal filling defect in the stent during the procedure, which resolved with intravenous abxicimab. There were no embolic or hemorrhagic complications in any patient. There were no deaths.

\section{Follow up}

Modified Rankin Scale was zero or one in all patients at discharge. Followup examinations have been done in 13 out of 16 patients till date. All patients who were followed up had an mRS of zero or one at 3 months. Three patients who underwent procedures recently are awaiting followup. Followup control angiograms of 13 patients done at 6 months duration did not show any regrowth or in-stent stenosis [Figures 1-4].

\section{Discussion}

The availability of neurovascular remodeling devices has opened new avenues for endovascular treatment of wide-necked and complex intracranial aneurysms. Earlier stents had various limiting characteristics such as inability to reposition once deployed, requirement of stiff delivery catheters, and poor visibility. ${ }^{[2]}$ The Solitaire AB stent is fully retrievable and can be redeployed to achieve optimal alignment across the neck of the aneurysm.

Studies have reported overall higher complication rates associated with stent-assisted coiling. ${ }^{[6,7]}$ The complications may be hemorrhagic (subarachnoid and lobar), thromboembolic (related to the stent), or technical (failure of delivery or other technical issues). ${ }^{[7]}$ These may be due to either the morphology of the aneurysm, difficulty in achieving complete occlusion, or as adverse effects of dual antiplatelet therapy.

There is sufficient literature regarding stent-assisted coiling using older stents. ${ }^{[8-11]}$ Only a few case series using the Solitaire stent have been published. ${ }^{[2,12]}$ Our study is the first series from India with Solitaire-assisted aneurysm coiling. We were able to achieve optimal placement of the stent across the aneurysm neck in all patients. There were no technical failures.

A literature survey of stent-supported coiling published recently reported initial complete occlusion rates of $45 \%$ with a complication incidence of $19 \%$ and death 


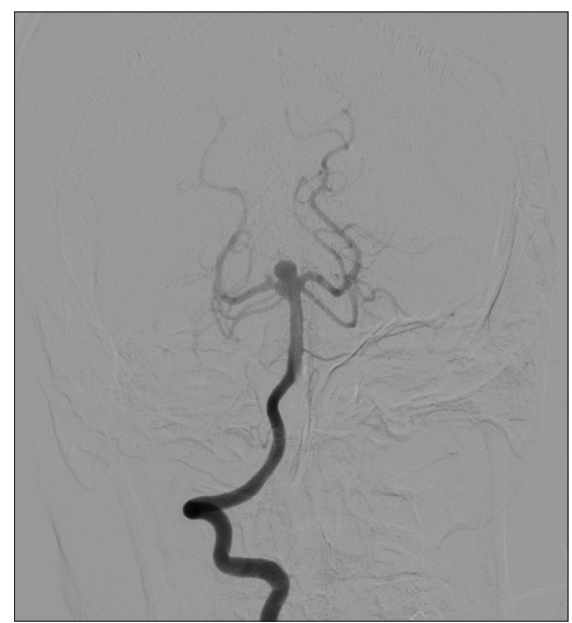

Figure 1: Right vertebral angiogram showing wide neck basilar top aneurysm with small right PCA aneurysm

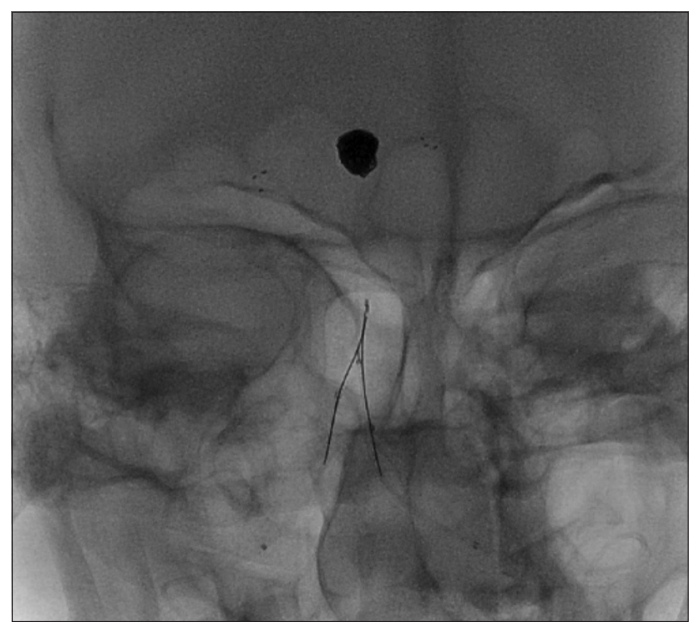

Figure 3: Fluoroscopic image showing $Y$ stent and coil mass

incidence of $2.1 \%{ }^{[7]} \mathrm{A}$ recent study from China using the Solitaire stent reported an initial complete occlusion rate of $83.9 \%$ with complications in six out of 31 patients and three deaths. ${ }^{[2]}$ We achieved complete occlusion in all patients with no morbidity and mortality.

\section{Conclusion}

In our experience, stent-assisted coiling using the Solitaire $A B$ stent is a safe and effective method of treating wide-necked and complex intracranial aneurysms. We agree that our study is small and although we encountered no morbidity and mortality in our patients, larger studies with long-term follow-ups are required to assess the efficacy and outcomes of stent-assisted aneurysm treatment. Newer generations of endovascular devices may further expand the effectiveness and scope of endovascular treatment.

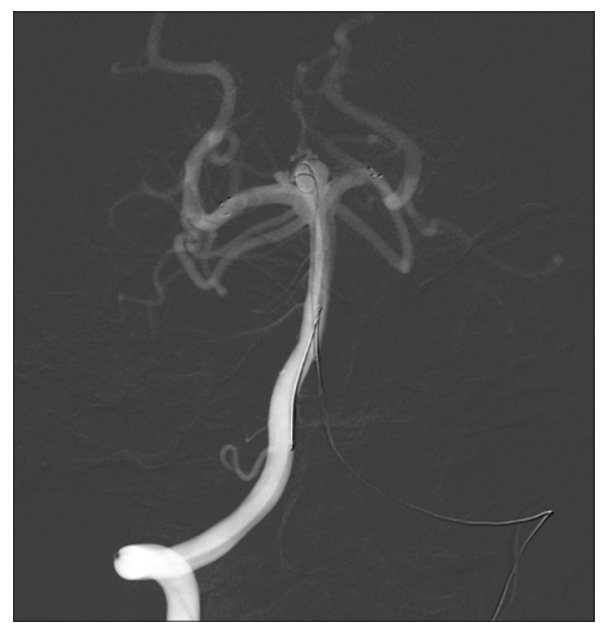

Figure 2: Road map showing coil deployment after $Y$ stenting

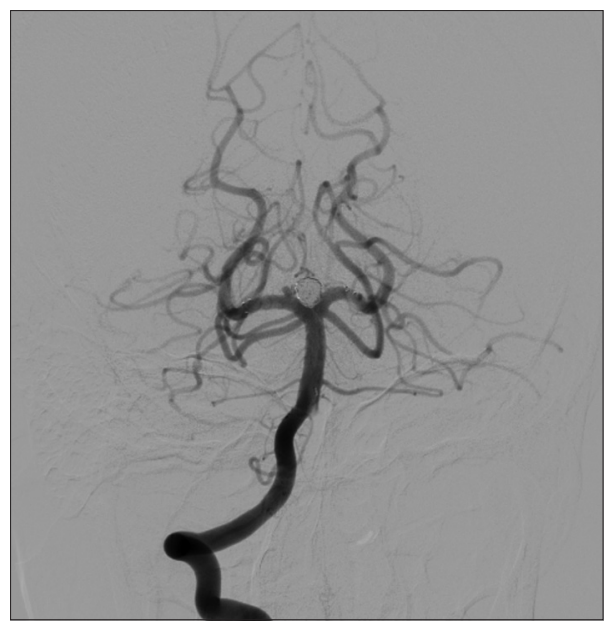

Figure 4: Postcoiling angiogram showing complete exclusion of basilar top aneurysm from circulation with patent bilateral posterior cerebral artery

\section{References}

1. Derdeyn CP, Barr JD, Berenstein A, Connors JJ, Dion JE, Duckwiler GR, et al. Executive Committee of the American Society of Interventional and Therapeutic Neuroradiology; American Society of Neuroradiology. The International Subarachnoid Aneurysm Trial (ISAT): A position statement from the Executive Committee of the American Society of Interventional and Therapeutic Neuroradiology and the American Society of Neuroradiology. AJNR Am J Neuroradiol 2003;24:1404-8.

2. Zhang J, Lv X, Yang J, Wu Z. Stent-assisted coil embolization of intracranial aneurysms using Solitaire stent. Neurol India 2012;60:278-82.

3. Spiotta AM, Wheeler AM, Smithason S, Hui F, Moskowitz S. Comparison of techniques for stent assisted coil embolization of aneurysms. J Neurointerv Surg 2012;4:339-44.

4. Van Swieten JC, Koudstaal PJ, Visser MC, Schouten HJ, van Gijn J. Interobserver agreement for the assessment of handicap in stroke patients. Stroke 1988;19:604-7.

5. Savio K, Pietra GL, Oddone E, Reggiani M, Leone MA. Reliability of the modified Rankin Scale applied by telephone. Neurol Int 2013;5:e2.

6. Bodily KD, Cloft HJ, Lanzino G, Fiorella DJ, White PM, Kallmes DF. Stent-assisted coiling in acutely ruptured intracranial aneurysms: A qualitative, systematic review of the literature. AJNR Am J Neuroradiol 2011;32:1232-6. 
7. Shapiro M, Becske T, Sahlein D, Babb J, Nelson PK. Stent-supported aneurysm coiling: A literature survey of treatment and follow-up. AJNR Am J Neuroradiol 2012;33:159-63.

8. Kim SR, Vora N, Jovin TG, Gupta R, Thomas A, Kassam A, et al. Anatomic results and complications of stent-assisted coil embolization of intracranial aneurysms. Interv Neuroradiol 2008;14:267-84.

9. Izar B, Rai A, Raghuram K, Rotruck J, Carpenter J. Comparison of devices used for stent-assisted coiling of intracranial aneurysms. PloS One 2011;6:e24875.

10. Ismail Alhothi A, Qi T, Guo S, Shi Z, Liang F, Yang L, et al. Neuroform stent-assisted coil embolization: A new treatment strategy for complex intracranial aneurysms. Results of medium length follow-up. Neurol Neurochir Pol 2010;44:366-74.

11. Raslan AM, Oztaskin M, Thompson EM, Dogan A, Petersen B, Nesbit G, et al. Neuroform stent-assisted embolization of incidental anterior communicating artery aneurysms: Long-term clinical and angiographic follow-up. Neurosurgery 2011;69:27-37.

12. Clajus C, Sychra V, Strasilla C, Klisch J. Stent-assisted coil embolization of intracranial aneurysms using the Solitaire ${ }^{\mathrm{TM}}$ AB Neurovascular Remodeling Device: Initial and midterm follow-up results. Neuroradiology 2013;55:629-38.

How to cite this article: Huded V, Nair RR, Vyas DD, Chauhan BN. Stent-assisted coiling of wide-necked intracranial aneurysms using the Solitaire AB stent. J Neurosci Rural Pract 2014;5:254-7.

Source of Support: Nil. Conflict of Interest: None declared.

\section{Author Help: Reference checking facility}

The manuscript system (www.journalonweb.com) allows the authors to check and verify the accuracy and style of references. The tool checks the references with PubMed as per a predefined style. Authors are encouraged to use this facility, before submitting articles to the journal.

- The style as well as bibliographic elements should be $100 \%$ accurate, to help get the references verified from the system. Even a single spelling error or addition of issue number/month of publication will lead to an error when verifying the reference.

- Example of a correct style

Sheahan P, O'leary G, Lee G, Fitzgibbon J. Cystic cervical metastases: Incidence and diagnosis using fine needle aspiration biopsy. Otolaryngol Head Neck Surg 2002;127:294-8.

- Only the references from journals indexed in PubMed will be checked.

- $\quad$ Enter each reference in new line, without a serial number.

- Add up to a maximum of 15 references at a time.

- If the reference is correct for its bibliographic elements and punctuations, it will be shown as CORRECT and a link to the correct article in PubMed will be given.

- If any of the bibliographic elements are missing, incorrect or extra (such as issue number), it will be shown as INCORRECT and link to possible articles in PubMed will be given. 